\title{
Behavioural coping patterns in Parkinson's patients with visual hallucinations
}

\author{
Jim Barnes ${ }^{\prime *}$, Vince Connelly', Laura Boubert ${ }^{2}$ and \\ Ksenija Maravic' \\ 'Department of Psychology, Oxford Brookes University, Oxford, UK \\ ${ }^{2}$ Department of Psychology, University of Westminster, London, UK
}

\begin{abstract}
Visual Hallucinations are considered to affect about $20 \%-40 \%$ of patients with Parkinson's disease. They are generally seen as a side effect of this long-term illness and can severely affect the daily quality of life of patients. The aim of this study was to determine the coping patterns or strategies used by patients and establish whether the phenomenology and behaviours used by patients enabled control of the phenomenon. Demographic and clinical variables were recorded, including motor measures, cognitive status, and depressive symptoms. Patient with hallucinations were at a more advance stage of the disease and displayed more depressive symptoms than their non-hallucinating counterparts. Most patients used more than one constructive coping strategy, the most common were simple behavioural strategies based around motor action or cognitive approaches resulting in visual modification. In addition, humour was a common technique used by the patients to deal with the phenomenon. Emotional responses varied between patients, but it was found that the actual content of the hallucination was not directly associated with whether it caused trouble to the patient, but perceived stress was strongly correlated with the subjective disturbing nature of visual hallucinations ( $\mathrm{VHs}$ ). This study gives insight into the role of cognitive-behavioural approaches when dealing with $\mathrm{VHs}$ and opens up avenues for future studies in helping patient to deal with hallucinations.
\end{abstract}

Parkinson's disease (PD), like many other long-term illnesses, has a profound effect on patients and their families and severely affects their ways of living (Lundqvist et al., 1997). As PD progresses the motor signs of PD worsen, medication treatment becomes less effective and with this comes increased dependency on carers and a decline in the patient's quality of life (Secker \& Brown, 2005). In addition to the motor complications, positive symptoms such as visual hallucinations (VHs) add to the hardship of illness leading to decreased life satisfaction and a shortened life expectancy (Inzelberg, Kipervasser, \& Korczyn, 1998). The motor and non-motor aspects of PD have led some to propose that the stress encountered in PD is multidimensional and multifaceted (Frazier,

*Correspondence should be addressed to Jim Barnes, Department of Psychology, Oxford Brookes University, Gipsy Lane, Oxford OX3 OBP, UK (e-mail jim.barnes@brookes.ac.uk). 
2000), with psychiatric symptoms such as VHs often outweighing the physical motor symptoms (Aarsland et al., 1999; Goetz, Leurgans, Pappert, Raman, \& Stemer, 2001). These non-motor symptoms may intensify anxiety level, disrupt daily life activities, and diminish self-esteem, and therefore gravely affect both the patient's and caregiver's quality of life (Diederich, Pieri, \& Goetz, 2003).

Although VHs are managed with antipsychotic medication, little is known about the behavioural management of VHs. Behavioural management of symptoms by the patient includes a range of coping strategies which are used frequently and efficiently in disorders accompanied by hallucinations (e.g., schizophrenia) and have been studied extensively in disorders related to stress (i.e., spinal cord injuries, cancer, and the carers of patients with a long-term illness; Carver, Scheier, \& Weintraub, 1989; Falloon \& Talbot, 1981; Lundqvist et al., 1997; Moorey, Frampton, \& Greer, 2003; Nayani \& David, 1996). In general, coping is defined as action-oriented and intrapersonal skills employed to manage external and internal demands and the perceived discrepancy between them (Lazarus \& Launier, 1978). It has been suggested that the use of appropriate coping strategies is a key factor in determining successful adjustment to severe physical illness/ disability (Lazarus \& Folkman, 1984). Effective coping strategies do not reduce the generation of negative thoughts, but rather encourage a set of skills that help people deal with the consequences of the symptoms when they occur (Barber \& DeRubeis, 1989). PD patients with VHs have been reported to use two main types of coping strategies: cognitive and interactive techniques, which are not related to the frequency of their hallucinations or their emotional response (Diederich et al., 2003). The Diedreich study restricted the questions to three main types of strategy (cognitive, interactive, and visual) and highlighted the need in future studies for an open-ended interview which would identify other effective coping strategies. Furthermore, future studies needed to address the relationship between these strategies and the patients' emotional response to hallucinations.

In summary, coping strategies have been shown to be effective in dealing with the stress of various symptoms in many different disorders, but as yet have not been fully examined in the area of VHs in PD. This study aimed to examine more closely the range of strategies employed by PD patients and then to investigate those most commonly used and those reported to be the most effective in terminating their hallucinations. Furthermore, we also wanted to assess if the patients' emotional response to their VHs modulates the extent to which the coping strategies are effective so as well as asking the patients about the level of stress they feel in relation to their hallucinations, this study also used a standardized measure of depression.

\section{Methods}

\section{Patients}

Respondents were recruited via a questionnaire as used in previous studies (Barnes \& David, 2001). All patients had a presumptive clinical diagnosis of PD. Participants were selected on the basis of experiencing VHs and were selected using a questionnaire asking about visual hallucinations and visual disturbances (Barnes \& David, 2001). Selection of individuals with visual hallucinations was based on (1) the frequency of hallucinations ( $>1$ per day); (2) the absence of epilepsy or cognitive impairment (MMSE $>25$; Folstein, Robins, \& Helzer, 1983). Although there is some evidence that cognitive impairment can lead to hallucinations (Manford \& Andermann, 1998), a cut-off of MMSE of 25 was chosen in this study as we sought only patients who could give a good account of their 
hallucinations. No participant had a clinical diagnosis of Alzheimer's disease or Lewy body dementia, or had any current or previous history of eye disease. The control patients with Parkinson's disease who had never hallucinated were matched with those with VHs for age and disease severity as well as levodopa medication dosage. No patient was taking any medication to control or alleviate their hallucinations.

\section{Assessments}

\section{Recruitment and coping strategies questionnaires}

The recruitment questionnaire was either mailed to participants or distributed at local Parkinson UK meetings in the Oxfordshire region. The questionnaire consisted of a typed A4 booklet and had a front page which contained information about the general nature of the study. The first section of the questionnaire was used to record the general demographics of the participants such as age and gender. A total of 168 questionnaires were distributed and 124 questionnaires were returned. Those indicating the presence of hallucinations in the past 3 months ( $n=34,27.4 \%)$ were asked to complete a second questionnaire on coping strategies and invited to attend a brief interview session. A control group was derived from those respondents who were non-hallucinators and who indicated a willingness to take part in the study $(n=31)$. The Hallucination Coping strategies questionnaire was constructed based on both personal communication with patients as well as on the literature review of coping strategies with VHs in PD (Diederich et al., 2003) and on coping with hallucinations in different disorders and illnesses (Carver et al., 1989; Lundqvist et al., 1997; Moorey et al., 2003). The questionnaire comprised items covering the nature of strategies used to cope with the hallucinatory experience and was derived from the seven main coping strategies believed to be employed by patients, namely relaxation, cognition, action, emotion, acceptance, religious, and humour. Patients were asked to think about and explain in as much detail as possible how they have coped with images, and circle how often they have used each method described. The answers varied from ' 3 - Regularly', 2 - 'Sometimes' to ' 1 - Not at all'. Patients were also asked if they could make the images appear (and if yes, how) and what other useful ways they could think of to make the images disappear (not stated in the questions).

As well as asking about the disturbance the VHs cause, the questionnaire also asked about the emotional response to the content of the hallucinatory experience. Based on the image content, the hallucinations reported were classified as objectively disturbing or non-disturbing. This categorization was related to previous reports of what constitutes an objectively disturbing image (Barnes \& David, 2001). Such images include dead people, spiders, rodents, or bizarre images which were unfamiliar. As well as this objective classification, patients were invited to give a subjective rating as to whether they found the images experienced disturbing and whether they or their carers found the occurrence of the hallucination stressful. Hallucinations were given a stress rating score between 1 and 3. ( 1 = not stressful, 2 = mildly stressful, and $3=$ very/moderately stressful $)$. A short cognitive screen including the mini mental state examination (MMSE) and the Beck depression inventory (BDI) were also administered.

\section{Demographics}

There were 36 patients ( 17 men) with, and 33 (14 men) without visual disturbances of any type. Those individuals who reported frequent migraines and ongoing eye disease ('hallucinators' $=2$, 'non-hallucinators' $=2$ ) were excluded. This left 34 coping 
Table I. Participant demographics

\begin{tabular}{|c|c|c|c|}
\hline & Hallucinators $(n=34)$ & Non-hallucinators $(n=3 \mathrm{I})$ & \\
\hline $\begin{array}{l}\text { Male } \\
\text { Female }\end{array}$ & $\begin{array}{l}23 \\
11\end{array}$ & $\begin{array}{l}20 \\
11\end{array}$ & $t(63)$ \\
\hline Age $($ year)* & $68.34(7.02)$ & $63.76(8.56)$ & 2.37 \\
\hline $\begin{array}{l}\text { Duration of } \\
\quad \text { illness (year)* }\end{array}$ & I I.34 (5.34) & $8.78(4.11)$ & 2.15 \\
\hline Daily levodopa (mg) & $598(176)$ & $679(169)$ & 1.87 \\
\hline Hoehn and Yahr* & $3.56(0.54)$ & $2.85(0.43)$ & 5.81 \\
\hline $\begin{array}{l}\text { Beck depression } \\
\text { inventory* }\end{array}$ & $18.67(4.5)$ & $16.30(5.0)$ & 2.01 \\
\hline MMSE & $26.8(1.5)$ & $27.3(1.2)$ & 1.47 \\
\hline
\end{tabular}

Note. ${ }^{*} p<.05$.

strategy questionnaires from hallucinators ( $23 \mathrm{men})$ and 31 from non-hallucinators (20 men) who completed the cognitive screening. The patient demographics are shown in Table 1.

\section{Procedure}

The study was introduced verbally and information sheets were given out at the monthly meetings in different PD societies throughout Oxfordshire. Potential participants with VHs who decided to take part contacted the experimenter at the end of the meeting. They were given an envelope with the coping strategies questionnaire to take home and complete over a period of 2 weeks, to be collected at the next meeting. Apart from the coping strategies questionnaires, all participants filled in a short questionnaire of the demographic variables.

\section{Results}

The two groups differed significantly on duration of illness $(t(63)=2.15, p<.05)$ and age $(t(63)=2.37, p<.05)$ with hallucinators being older and having a longer disease duration, but did not differ in daily levodopa medication $(t(63)=1.87, p>.05)$ or MMSE $(t(63)=1.47, p>.05)$. The hallucinators also had greater disease severity as measured by the Hoehn \& Yahr $(t(63)=5.81, p<0.05)$. These results indicate that the clinical presentation of patients was similar to other studies.

\section{Coping strategies}

The aim of this investigation was to examine the range and type of coping strategies in PD patients who experience regularVHs. The patients were asked to report all strategies used and detail where multiple strategies were used. Of the 34 patients, only 10 (29\%) believed they could control their hallucinatory experience in some way. The majority of patients had accepted that their hallucinations come and go and as such acceptance was a commonly reported behaviour with 16 patients (47\%) regularly not performing any effortful strategy to extinguish their hallucinations. The most frequently used coping 
strategies included a cognitive approach $(n=18,53 \%)$ where patients tried to rationalize the events by the application of cognitive effort to tell themselves the images were not real and to inspect the images to gain more detail about them. Emotion coping was used by 14 patients (41\%), here talking and discussing their feeling of fear with somebody relieved the emotional burden of the experience. Other patients $(n=22,65 \%)$ indicated that they took action about their hallucinations like rubbing their eyes or turning on the lights and even trying to talk or interact with the images they saw. Humour featured as a way patients deal with the phenomenon, as 12 patients (35\%) described that the best way to deal with the VHs and indeed the disorder of PD was to make jokes about it to family and friends. In the final question they were asked about which single strategy they believe was the most effective. Action-oriented strategies were believed to be the most effective with 20 patients (59\%) indicating that it was their strategy of choice. The second most effective single strategy used was cognitive $(n=8,24 \%)$, followed by emotional ( $n=4,12 \%)$ while four patients believed that there was really no point in having any strategy $(n=4,12 \%)$. Examples of coping strategies and the total number of responses in each category from patients are shown in Table 2.

\section{Emotional response to $\mathrm{VHs}$}

Depression scores indicated a significant difference whereby the hallucinator group was more depressed than the non-hallucinator group $(t(63)=2.01, p<0.05)$. Patients' and carers' independent rating of the amount of stress the hallucinations caused them highlighted that carers found the VHs more distressing than the patients with $85 \%$ of carers compared with $45 \%(n=16)$ of patients finding them very stressful or moderately stressful. Some patients $(n=12,35 \%)$ expressed that the greatest cause of worry was the reaction of their carer to the VHs.

The phenomenological content of the hallucination could not be simply associated with whether it caused disturbance to the patient. Of the 34 patients, $n=14$ (41\%) found their hallucinations subjectively disturbing, but the images were not always objectively disturbing. Indeed there was no correlation between subjective and objective ratings of the disturbing nature of the VHs and most of the patients who were disturbed by their images had benign or unspecific content in their hallucination. However, the perceived stress was strongly correlated with the subjective disturbing nature of VHs. See Table 3.

\section{Discussion}

The sample in this study might not be fully representative of the whole population that have PD and VHs and the recruitment procedures might have caused a self-selecting bias within the groups. Nevertheless, the recruited patients were willing to describe their experiences of VHs and as a result some general patterns of coping strategies were discernible. Except for two patients, each patient used more than one constructive coping strategy. Simple behavioural strategies (either action-oriented or cognitive approaches), mainly based on visual modifications (e.g., rubbing the eyes, blinking, turn the lights on) were reported as the most efficient. The most common way previously reported of interacting with the images was by walking towards them or by trying to touch them (Barnes \& David, 2001). Similar interactive techniques, where the patients verify the nonreality of the hallucinations have also been reported as a common strategy (Diederich et al., 2003). Interestingly in this study, only five patients reported touching the images as an efficient mean of coping with VHs, but instead they used more remote techniques to 
Table 2. Example of coping strategies and the total number of responses in each category from patients

\begin{tabular}{|c|c|c|c|}
\hline Coping strategy & Example & Number & Percentage \\
\hline Action-oriented Approach & $\begin{array}{l}\text { I blink and they go away or change } \\
\text { I try and touch the image } \\
\text { I rub my eyes } \\
\text { I turn on the lights } \\
\text { I watch TV }\end{array}$ & 22 & 65 \\
\hline Cognitive & $\begin{array}{l}\text { I am aware that they are not really there } \\
\text { I just understand that the images are not real } \\
\text { I know my brain just misinterprets things } \\
\text { I think about the nature of the image } \\
\text { I try and work out why I see these things }\end{array}$ & 18 & 53 \\
\hline Emotional & $\begin{array}{l}\text { I talk things over with someone } \\
\text { I talk about my hallucinations } \\
\text { I try and get people to understand my fears } \\
\text { I get upset by my images } \\
\text { They make me very emotional }\end{array}$ & 14 & 41 \\
\hline Religious & $\begin{array}{l}\text { I ask god for help } \\
\text { It is a divine gift } \\
\text { I just know it is a punishment for something } \\
\text { I pray they go away } \\
\text { I trust in god }\end{array}$ & 8 & 24 \\
\hline Humour & $\begin{array}{l}\text { I laugh about them } \\
\text { I make jokes about them } \\
\text { I try and see the funny side of it } \\
\text { I tell funny stories about them } \\
\text { The whole thing is a bit of a joke }\end{array}$ & 12 & 35 \\
\hline Acceptance & $\begin{array}{l}\text { I just wait until they disappear } \\
\text { I think it is just part of the disorder } \\
\text { The doctors can't stop them } \\
\text { I just have to live with them } \\
\text { No point in trying to fight it }\end{array}$ & 12 & 35 \\
\hline No coping strategy & $\begin{array}{l}\text { I do not do anything } \\
\text { There is no point in trying to control them } \\
\text { It is futile to try } \\
\text { I feel I have no control } \\
\text { I cannot alter my perception }\end{array}$ & 16 & 47 \\
\hline
\end{tabular}

Table 3. Spearman rho correlations between stress, control, and disturbance

\begin{tabular}{llcc}
\hline & Stress & Control & Disturbing (S) \\
\hline Control & 0.173 & & \\
Disturbing (S) & $0.865^{* *}$ & 0.132 & \\
Disturbing (O) & -0.034 & -0.134 & -0.112 \\
\hline
\end{tabular}

Note. ${ }^{* *} p<.01$.

control their experiences. Acceptance was also a commonly reported strategy to extinguish hallucinations. This strategy was not associated with the phenomenological content of the hallucination, but rather with the frequency with which the patient 
was experiencing the phenomenon. The more hallucinations the patient experienced during the day the less willing they were to interact with them, primarily as a result of physical tiredness and general decline in cognitive vigour.

Dopaminergic over-stimulation of retinal neurons have been related to partial sensory deprivation (Diederich et al., 2003), which is a long-appreciated risk factor for hallucinations in other clinical contexts (e.g., Charles Bonnet syndrome; Teunisse, Cruysberg, Hoefnagels, Verbeek, \& Zitman, 1996) as it permits the (re)emergence or release of previously recorded percepts (Diederich, Goetz, \& Stebbins, 2005). PD patients from this study frequently reported lightening the environment (e.g., turning the lights on) as a way of stopping the hallucinations. In accordance with the results from the study on the nature of VHs in PD, this coping strategy further clarifies the importance of a dimly lit environment as an important predisposition for the generation of VHs. Similarly, closing the eyes (or just blinking) and focusing on the images were described as a frequent coping strategy. It is possible that through these strategies PD patients improve their visual acuity, a factor proposed to be critical in the generation of VHs (Büttner et al., 1996; Diederich et al., 1998; Menon, 2005). Further support for this hypothesis is provided by earlier findings that some patients report that their VHs are peripheral and that they disappear when they concentrate on them (Barnes \& David, 2001; Diederich et al., 2003).

Most coping strategies combined practical action-oriented behavioural and cognitive components to enhance understanding. This combination is a basis for cognitivebehavioural therapy, which aims to develop both cognitive and behavioural skills to cope with stressors, and is beneficial not only for the patients with long-term illnesses but also for their carers (Starker \& Jolin, 1982). The use of active coping strategies is also in line with the notion that problem-focused, active coping may be best for behavioural change, and emotional regulation may be best for psychological reactions to illness (Frazier, 2000). Furthermore, emotional coping may be more efficient when dealing with the ambiguity and unpredictability of illness (Maes, Leventhal, \& De Ridder, 1996). There was a leaning towards the notion that VHs might cause depression in PD and this may again be a significant contributor to the experience. A mild-moderate depression in PD patients with VHs is most likely as a result of having the illness, but it may also exacerbate the experience of not being in control of one's perceptions and lead to undue stress. This is supported by the finding that 16 patients reported being stressed over their VHs in the past month and of these 11 patients were in the moderate depression level as measured by the BDI and one patient was in the severe range. It has been proposed that hallucinations become distressing only when appraised as uncontrollable and dangerous (Morrison, Wells, \& Nothard, 2000). However, the control over images was not related to how stressed patients felt about their VHs, instead the level of stress about VHs seemed to be related to how disturbing the images were for the patients themselves that is, the subjective perception of the individual and had no relation to the actual images themselves which in many cases seemed to be unthreatening or even unspecific. Therefore, the level of stress is very subjective and independent of hallucination management and the usefulness of coping strategies used.

In conclusion, this study gives insight into the role of cognitive-behavioural approaches when dealing with VHs. PD patients with VHs from our study have most commonly used a combination of action-oriented and cognitive coping strategies. Depression in PD is in all probability related to the disease itself and not to the VHs, but may have some impact on patients in establishing and maintaining control of their perceptions. Looking at the content of the hallucinatory experience, the stressful nature of the VHs is surprisingly not related to the voluntary control exhibited over the images, 
but more associated with the subjective phenomenological content of the phenomenon. While visual hallucinations may not always upset the patient or their carer, they are always very real. Hence, the introduction of coping techniques and behavioural strategies may help patients to react when hallucinations manifest themselves and ultimately increase their quality of life.

\section{References}

Aarsland, D., Larsen, J. P., Lim, N. G., Janvin, C., Karlsen, K., Tandberg, E., \& Cummings, J. L. (1999). Range of neuropsychiatric disturbances in patients with Parkinson's disease. Journal of Neurology Neurosurgery \& Psychiatry, 67, 492-496. doi:10.1136/jnnp.67.4.492

Barber, J. P., \& DeRubeis, R. J. (1989). On second thought: Where the action is in cognitive therapy for depression. Cognitive Therapy and Research, 13, 441-457. doi:10. 1007/BF01173905

Barnes, J., \& David, A. S. (2001). Visual hallucinations in Parkinson's disease: A review and phenomenological survey. Journal of Neurology Neurosurgery \& Psychiatry, 70, 727-733. doi:10.1136/jnnp.70.6.727

Büttner, T., Kuhn, W., Müller, T., Welter, F. L., Federlein, J., Heidbrink, K., \& Przuntek, H. (1996). Visual hallucinosis: The major clinical determinant of distorted chromatic contour perception in Parkinson's disease. Journal of Neural Transmission, 103, 1195-1204. doi:10. 1007/BF01271204

Carver, C. S., Scheier, M. F., \& Weintraub, J. K. (1989). Assessing coping strategies: A theoretically based approach. Journal of Personality \& Social Psychology, 56, 267-283. doi:10.1037//0022-3514.56.2.267

Diederich, N. J., Goetz, C. G., Raman, R., Pappert, E. J., Leurgans, S., \& Piery, V. (1998). Poor visual discrimination and visual hallucinations in Parkinson's disease. Clinical Neuropharmacologl, 21, 289-295.

Diederich, N. J., Goetz, C. G., \& Stebbins, G. T. (2005). Repeated visual hallucinations in Parkinson's disease as disturbed external/internal perceptions: Focused review and a new integrative model. Movement Disorders, 20, 130-140. doi:10.1002/mds.20308

Diederich, N. J., Pieri, V., \& Goetz, C. G. (2003). Coping strategies for visual hallucinations in Parkinson's disease. Movement Disorders, 18, 831-832. doi:10.1002/mds.10450

Falloon, I. R. H., \& Talbot, R. E. (1981). Persistent auditory hallucinations - coping mechanisms and implications for management. Psychological Medicine, 11, 329-339. doi:10.1017/ S0033291700052144

Folstein, M. F., Robins, L. N., \& Helzer, J. E. (1983). The mini-mental state examination. Archives of General Psychiatry, 40, 812.

Frazier, L. D. (2000). Coping with disease-related stressors in Parkinson's disease. Gerontologist, 40, 53-63. doi:10.1093/geront/40.1.53

Goetz, C. G., Leurgans, S., Pappert, E. J., Raman, R., \& Stemer, A. B. (2001). Prospective longitudinal assessment of hallucinations in Parkinson's disease. Neurology, 57, 2078-2082. doi:10.1212/WNL.57.11.2078

Inzelberg, R., Kipervasser, S., \& Korczyn, A. D. (1998). Auditory hallucinations in Parkinson's disease. Journal of Neurology Neurosurgery \& Psychiatry, 64, 533-535. doi:10.1136/jnnp.64.4.533

Lazarus, R. S., \& Folkman, S. (1984). Stress, appraisal, and coping. New York: Springer.

Lazarus, R. S., \& Launier, R. (Eds.). (1978). Stress-related transactions between person and environment. New York: Plenum.

Lundqvist, C., Siosteen, A., Sullivan, L., Blomstrand, C., Lind, B., \& Sullivan, M. (1997). Spinal cord injuries: A shortened measure of function and mood. Spinal Cord, 35, 17-21. doi:10.1038/sj.sc.3100347 
Maes, S., Leventhal, H., \& de Ridder, D. T. D. (1996). Coping with chronic disease. In M. Zeidner \& N. S. Endler (Eds.), Handbook of Coping. Theory, research, applications (pp. 221-251). New York: Wiley.

Manford, M., \& Andermann, F. (1998). Complex visual hallucinations. Clinical and neurobiological insights. Brain, 121, 1819-1840. doi:10.1093/brain/121.10.1819

Menon, G. J. (2005). Complex visual hallucinations in the visually impaired: A structured history-taking approach. Archives of Ophthalmology, 123, 349-355. doi:10.1001/archopht. 123.3 .349

Moorey, S., Frampton, M., \& Greer, S. (2003). The cancer coping questionnaire: A self-rating scale for measuring the impact of adjuvant psychological therapy on coping behaviour. Psychooncology, 12, 331-344. doi:10.1002/pon.646

Morrison, A. P., Wells, A., \& Nothard, S. (2000). Cognitive factors in predisposition to auditory and visual hallucinations. British Journal of Clinical Psychology, 39, 67-78. doi:10.1348/ 014466500163112

Nayani, T. H., \& David, A. S. (1996). The auditory hallucination: A phenomenological survey. Psychological Medicine, 26, 177-189. doi:10.1017/S003329170003381X

Secker, D. L., \& Brown, R. G. (2005). Cognitive behavioural therapy (CBT) for carers of patients with Parkinson's disease: A preliminary randomised controlled trial. Journal of Neurology Neurosurgery \& Psychiatry, 76, 491-497. doi:10.1136/jnnp.2004.042291

Starker, S., \& Jolin, A. (1982). Imagery and hallucination in schizophrenic patients. Journal of Nervous \& Mental Disease, 170, 448-451. doi:10.1097/00005053-198208000-00002

Teunisse, R. J., Cruysberg, J. R., Hoefnagels, W. H., Verbeek, A. L., \& Zitman, F. G. (1996). Visual hallucinations in psychologically normal people: Charles Bonnet's syndrome. Lancet, 347, 794-797. doi:10.1016/S0140-6736(96)90869-7

Received 29 June 2012; revised version received 6 February 2013 